\title{
Determinación de residuos de abamectina-triclabendazol en tejidos bovinos ${ }^{\#}$
}

\author{
Determination of abamectin-triclabenzadole residues in bovine tissues \\ C Palma ${ }^{1}$, C Godoy ${ }^{1}$, M Arboix ${ }^{2}$, R Pérez $^{*}$ \\ ${ }^{1}$ Laboratorio de Farmacología, Facultad de Medicina Veterinaria, Universidad de Concepción, Campus Chillán, Chile. \\ ${ }^{2}$ Departamento de Farmacología y Toxicología, Facultad de Medicina Veterinaria, Universidad Autónoma de Barcelona, España.
}

\begin{abstract}
SUMMARY
Abamectin (ABM) and Triclabendazole (TCBZ) are two potent antiparasitic drugs that differ in their chemical structure and spectrum of action. In this study, a sensitive HPLC-method was developed and validated to determine ABM and TCBZ residues in bovine edible tissues, and the pattern of tissue depletion of an oral formulation containing the association abamectin (ABM) - triclabendazole (TCBZ) was characterized. Sixteen parasitefree heifers ( $232 \pm 37.5 \mathrm{~kg}$ body weight) were treated with the oral formulation at a dose rate of $0.2 \mathrm{mg}$ of ABM/kg and $10 \mathrm{mg}$ of TCBZ/kg of live weight. Treated animals were slaughtered weekly in randomly selected groups of three to four animals from day 7 until day 42 post-treatment. Concentration profiles of the drugs were determined in liver, kidney, muscle and fat tissues. Two nontreated animals were slaughtered in order to obtain blank tissue samples to validate the analytical methodology. Drug concentration profiles were determined by a validated HPLC analytical methodology using fluorescence detection for ABM and UV- detection for TCBZ. The highest concentration of ABM was observed in liver samples $(4.02 \mathrm{ng} / \mathrm{g})$ and was maintained for 14 days. In the case of $\mathrm{TCBZSO}_{2}$ the highest concentration was observed in the kidney $(0.79 \mu \mathrm{g} / \mathrm{g})$ and persisted for a period of 14 days.
\end{abstract}

Palabras clave: abamectina, triclabendazol, HPLC, residuos tisulares.

Key words: abamectin, triclabendazole, HPLC, tissue residues.

\section{INTRODUCCION}

El desarrollo de nuevos antihelmínticos para el control del parasitismo en las diferentes especies domésticas ha sido el área donde la investigación farmacéutica ha experimentado la mayor expansión durante los últimos años. El amplio uso de estos compuestos y la necesidad de asegurar la calidad e inocuidad de los alimentos provenientes de animales tratados con antihelmínticos, ha llevado a desarrollar técnicas y metodologías cada vez más sensibles y exactas para la determinación de estos fármacos en diferentes tejidos animales. La cromatografía líquida de alta eficiencia (HPLC) en fase reversa representa una técnica analítica confiable y de alta precisión, mediante la cual es posible estudiar el perfil de depleción de residuos de dos antiparasitarios de acción complementaria como son abamectina (ABM) y triclabendazol (TCBZ).

$\mathrm{ABM}$ es una lactona macrocíclica perteneciente al grupo de las avermectinas (AVM), que son una serie de compuestos naturales y semisintéticos, que poseen un mecanismo de acción común, con potente actividad

\footnotetext{
Aceptado: 21.03.2006.

Financiado por Laboratorio Intervet Veterinaria Chile.

\# Proyecto: PI 202.151.012-1.3 Dirección de Investigación Universidad de Concepción.

*rubperez@udec.cl
}

antihelmíntica y endectocida (McKellar y Benchaoui 1996).

El TCBZ (5-cloro-6-(2,3-diclorofenoxi)-2-metiltiobencimidazol) es un bencimidazol halogenado que posee elevada actividad frente a estados inmaduros y adultos de Fasciola hepática, habiendo demostrado una elevada eficacia fasciolicida en ovinos, caprinos y bovinos (Misra y col 1987, Wolff y col 1983). Esta acción se asocia principalmente con el metabolito activo triclabendazol sulfóxido (TCBZSO), ya que el fármaco original TCBZ es rápidamente metabolizado en el hígado hacia sulfóxido y posteriormente a sulfona (TCBZSO ${ }_{2}$ ), el cual es inactivo (Hennessy y col 1987).

En las prácticas de manejo intensivo en la crianza de animales, la asociación de fármacos antiparasitarios para lograr un espectro antihelmíntico más amplio, es frecuentemente utilizada por los ganaderos tanto para tratamientos preventivos como curativos de las parasitosis. Así la asociación de ABM y TCBZ es una formulación comercial que ha sido diseñada para entregar una protección adecuada contra nemátodos, artrópodos y tremátodos.

Por constituir una formulación farmacéutica de aparición reciente en el mercado nacional, no existen antecedentes acerca de sus concentraciones residuales en tejidos de consumo para estos fármacos cuando se administran asociados, por lo que el objetivo de este estudio fue determinar la presencia y persistencia de residuos de ABM y TCBZ o sus principales metabolitos en tejidos comestibles, tales como músculo, hígado, riñón y grasa, 
posterior a la administración de una formulación para uso oral en bovinos.

\section{MATERIAL Y METODOS}

Se trabajó con un grupo de 16 vaquillas de raza Holstein Friesian con un peso promedio de $232 \pm 37,5 \mathrm{~kg}$. A cada animal se le administró un volumen de producto equivalente a una dosis de $0,2 \mathrm{mg} / \mathrm{kg}$ de ABM y de $10 \mathrm{mg} / \mathrm{kg}$ de TCBZ vía oral (Sofomax ${ }^{\circledR}$, Intervet Veterinaria Chile Ltda.). Para determinar los niveles de residuos de estos fármacos en tejidos comestibles, grupos de 3 a 4 animales fueron sacrificados a $\operatorname{los} 7,14,28$ y 42 días postratamiento y enviados a una planta faenadora, donde se recolectaron las muestras respectivas desde hígado, riñón, grasa y músculo.

Descripción de los procedimientos analíticos. ABM, TCBZ y sus metabolitos triclabendazol sulfóxido (TCBZSO) y triclabendazol sulfona $\left(\mathrm{TCBZSO}_{2}\right)$ fueron separados a través de procedimientos de extracción en fase sólida y determinados mediante cromatografía líquida de alta resolución (HPLC) con detección por fluorescencia en el caso de abamectina y detección ultravioleta para triclabendazol.

\section{ABAMECTINA}

Rectas de calibración. Muestras de tejido blanco, procedentes de animales no tratados: hígado, riñón, músculo (1g) y grasa $(0,1 \mathrm{~g})$ fueron sobrecargadas con ABM (pureza patrón de ABM 96,0\%, Lote 990810, Laboratorio Intervet, Chile, Ltda.), para obtener las concentraciones finales de 0,5-1-2-5-10 y 25 ng/g. Las soluciones estándar fueron preparadas cada tres meses, almacenadas en refrigeración $\left(2\right.$ a $\left.8^{\circ} \mathrm{C}\right)$ y equilibradas a temperatura ambiente antes de su uso.

Procesamiento. Las muestras de tejido sobrecargado (hígado, riñón, músculo y grasa) y las muestras problema fueron sometidas a una extracción con solventes y posteriormente a extracción en fase sólida (EFS), según la metodología descrita por Lifschitz y col (2000).

Procedimiento de derivatización. El eluido de cada muestra se evaporó bajo flujo continuo de nitrógeno en un baño termorregulado (Memmert Modelo DIN12877-KI, Alemania) a $60^{\circ} \mathrm{C}$. El residuo seco fue disuelto en $100 \mu \mathrm{L}$ de una solución de N-Metilimidazol (Sigma-Aldrich, USA) en acetonitrilo (1:1 v/v) y $150 \mu \mathrm{L}$ de una solución de anhídrido trifluoroacético (Sigma-Aldrich, USA) en acetonitrilo (1:2 v/v) (Payne y col 1995).

Condiciones Cromatográficas. La fase móvil estuvo compuesta por una mezcla de ácido acético (0,2\%)metanol-acetonitrilo-(2:34:64 v/v), bombeada a flujo de
1,5 mL/min. a través de una columna Supelclean LC-18 Supelco, Bellefonte, USA. (5 $\mu \mathrm{m} ; 4,6$ mm x $150 \mathrm{~mm}$ ). Se empleó un sistema de detección por fluorescencia a una longitud de onda de excitación 383 nm y 447 nm de emisión. Para establecer el tiempo de retención del fármaco, $10 \mu \mathrm{L}$ de una solución estándar de $1 \mathrm{ng} / \mu \mathrm{L}$ de ABM fue inyectada en el sistema cromatrográfico, obteniéndose un tiempo de 7,8 minutos.

\section{TRICLABENDAZOL}

Rectas de calibración: Muestras de tejido, hígado, riñón, músculo ( $1 \mathrm{~g})$ y grasa $(0.1 \mathrm{~g})$ procedentes de animales no tratados, fueron sobrecargadas con TCBZ y sus metabolitos sulfóxido y sulfona para obtener concentraciones de 0,$05 ; 0,1 ; 0,5 ; 1,0 ; 5,0 ; 10$ y $15 \mu \mathrm{g} / \mathrm{g}$, agregando, además, $1 \mu \mathrm{g}$ de oxfendazol (OXF) como estándar interno. Los patrones de TCBZ (pureza: 99,7\%), TCBZSO (pureza: 93,7\%) y TCBZSO $_{2}$ (pureza: 93,7\%) y el estándar interno de OXF (pureza: 99,2\%) fueron diluidos en metanol.

Procesamiento. Las muestras de tejido sobrecargado y las muestras problema fueron sometidas a una extracción con solventes, y luego a extracción en fase sólida (EFS), según procedimientos descritos por Alvinerie y col (1996).

Condiciones Cromatográficas. La fase móvil estuvo compuesta por una mezcla de acetonitrilo-acetato de amonio 0,025 M pH:6.6 (60:40 v/v), bombeada a flujo de $1,2 \mathrm{~mL} / \mathrm{min}$. a través de una columna Supelclean LC-18, Supelco, Bellefonte, USA. (5 $\mu \mathrm{m} ; 4,6 \mathrm{~mm}$ x $250 \mathrm{~mm}$ ), y se empleó un sistema de detección por luz ultravioleta (UV) a longitud de onda de $300 \mathrm{~nm}$ (Detector SPD10Avp, Shimadzu). Soluciones estándar de trabajo fueron inyectadas en el sistema cromatrográfico para determinar los tiempos de retención de OXF, TCBZ y sus metabolitos, los que fueron de 2,5 min para OXF; 12,9 min para TCBZ; 4,5 min para el metabolito $\mathrm{TCBZSO}_{2}$ y 5,83 min para el metabolito TCBZSO.

Parámetros de Validación de la Metodología Analítica. La validación de los métodos analíticos se realizó según las recomendaciones de EMEA (1998) y Shah (1992) en muestras en quintuplicado en las que se determinó; la especificidad, la recuperación, la linealidad, el límite de cuantificación y el límite de detección.

La especificidad se determinó comparando cromatogramas de muestras de tejido libre de fármaco con cromatogramas de muestras sobrecargadas con ABM o TCBZ según corresponda.

La recuperación del fármaco se calculó estableciendo la relación entre las áreas de los picos cromatográficos, existente en las muestras de tejido sobrecargadas con concentraciones conocidas del analito en estudio, con las obtenidas de la inyección directa del estándar puro di- 
suelto en un solvente apropiado. Los resultados se expresan en porcentajes $(\%)$.

La linealidad fue determinada por inyección de muestras de tejido sobrecargadas con el analito en estudio en concentraciones crecientes y en quintuplicado. Las rectas de calibración fueron obtenidas mediante un análisis de regresión, representando las concentraciones de fármaco en el eje de las abscisas y el área de los picos cromatográficos en el de las ordenadas.

El límite de cuantificación (L.C.) se determinó a partir de la respuesta del blanco y se calculó como el promedio del intercepto (b) de las rectas de calibración más 10 veces su desviación estándar y dividido por el valor promedio de la pendiente (a) de las mismas rectas de calibración (Prieto y col 2003, Quattrocchi 1992).

$$
\text { L.C. }=\left(\text { Promedio }_{\mathrm{b}}+10 \times\left(\text { D.E }_{\mathrm{b}}\right) / \mathrm{a}\right.
$$

El límite de detección (L.D.) se determinó a partir de la respuesta del blanco y se calculó como el promedio del intercepto de las rectas de calibración más 3 veces su desviación estándar y dividido por el valor promedio de la pendiente de las mismas rectas de calibración (Prieto y col 2003, Quattrocchi 1992).

$$
\text { L.D. }=\left(\text { Promedio }_{\mathrm{b}}+3 \times\left(\text { D.E. }_{\mathrm{b}}\right) / \mathrm{a}\right.
$$

Análisis estadístico. El cálculo de los parámetros estadísticos: promedio, desviación estándar (DE) coeficiente de variación $(\mathrm{CV})$ y error medio o relativo (EM) fue realizado mediante el programa Microsoft Excel 97 (Microsoft Office 97, Microsoft Corporation).

\section{RESULTADOS Y DISCUSION}

\section{VALIDACION DE LA METODOLOGIA ANALITICA}

Abamectina. Un cromatograma típico de la solución estándar de 10 ng de ABM (externo) y los cromatogramas de tejido blanco y tejido sobrecargado con el fármaco (interno), se muestran en la figura 1. La ausencia de interferencias en la separación sugiere una alta especificidad del método cromatográfico y una buena selectividad del procedimiento de extracción (Roudaut 1998).

La metodología aplicada para extracción de ABM desde tejidos permitió obtener adecuados porcentajes de recuperación (cuadro 1), los que fueron similares a los obtenidos para ABM y para otras lactonas macrocíclicas, como son ivermectina, doramectina, eprinomectina y moxidectina, en un estudio multirresidual realizado en hígado de bovinos, mediante cromatografía líquida con detección por fluorescencia (Ali y col 2000). Se describe que el hígado es uno de los tejidos más difíciles de tratar,
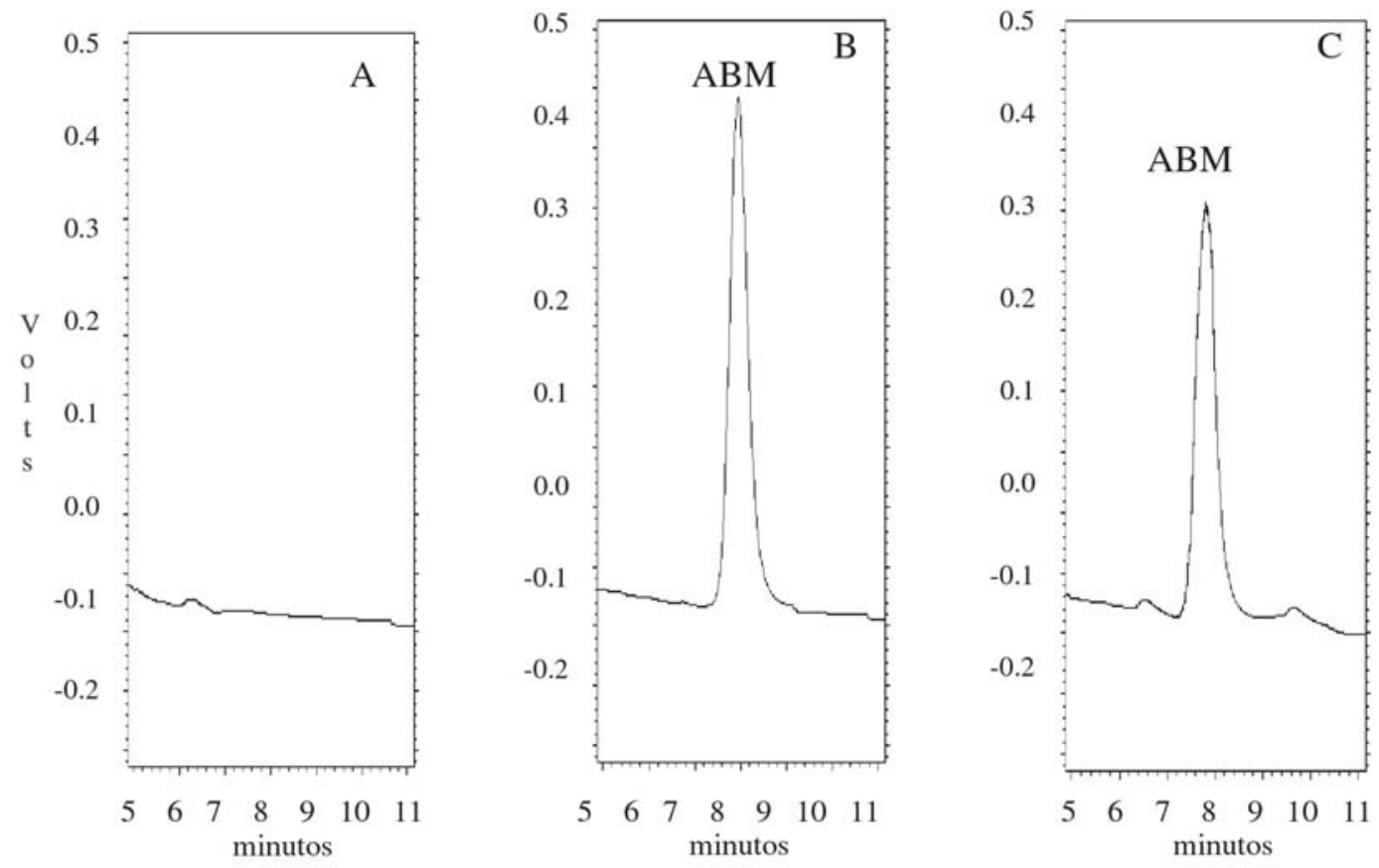

Figura 1. Cromatograma blanco de hígado bovino (A). No se observan interferencias en el tiempo de retención del fármaco en análisis. Cromatograma típico de solución patrón de 10 ng de abamectina (B). Cromatograma de hígado de bovino sobrecargado con $10 \mathrm{ng} / \mathrm{g}$ de abamectina (C).El tiempo de retención para abamectina fue 7,8 minutos.

Chromatogram of bovine liver blank (A). No interferences in the retention time for the drug was observed. Typical chromatogram of a standard solution of $10 \mathrm{ng}$ of abamectin (ABM) (B). Chromatogram of bovine liver fortified with $10 \mathrm{ng} / \mathrm{g}$ of abamectin $(\mathrm{ABM})(\mathrm{C})$. The retention time for abamectin was 7,8 minutes. 
Cuadro 1. Parámetros de validación de la metodología analítica para la determinación de las concentraciones de abamectina (ABM) en tejidos comestibles de bovino.

Analytical methodology validation for the determination of abamectin concentrations in edible bovine tissues.

\begin{tabular}{lcccc}
\hline & \multicolumn{4}{c}{ Tejidos } \\
\cline { 2 - 5 } $\begin{array}{l}\text { Parámetros } \\
\text { de validación }\end{array}$ & Hígado & Riñón & Músculo & Grasa \\
\hline $\begin{array}{l}\text { Porcentaje } \\
\text { de recuperación (\%) }\end{array}$ & 78,00 & 79,60 & 80,95 & 84,21 \\
$\begin{array}{l}\left.\text { Linealidad (r }{ }^{2}\right) \\
\text { Precisión (C.V.) (\%) }\end{array}$ & 0,9964 & 0,9977 & 0,9980 & 0,9972 \\
$\begin{array}{l}\text { Exactitud (E.M.) (\%) } \\
\text { Límite de }\end{array}$ & 14,128 & 4,451 & 12,450 & 2,319 \\
cuantificación (ng/g) & 0,528 & 0,550 & 0,142 & 3,202 \\
$\begin{array}{l}\text { Límite de } \\
\text { detección (ng/g) }\end{array}$ & 0,283 & 0,232 & 0,088 & 0,789 \\
\hline
\end{tabular}

$\mathrm{r}^{2}$ : coeficiente de determinación; \%: porcentaje; C.V.: coeficiente de variación; E.M.: error medio; ng/g: nanogramos por gramo.

debido a la gran cantidad de interferencias generadas por productos de degradación hepática; por lo tanto, la detección de un analito en este tejido se utiliza como referencia para evaluar la metodología de extracción aplicada a otros tejidos como, por ejemplo, músculo, grasa, leche, entre otros (Wu y col 2001).
La linealidad de los procedimientos analíticos para ABM estableció coeficientes de determinación que fueron superiores a 0,990 , lo cual indica que existe una relación directamente proporcional entre la respuesta del detector (valor de área) y la cantidad teórica correspondiente a la concentración de analito presente en el tejido. Los valores de coeficientes de variación (cuadro 1) en los tejidos estudiados fueron inferiores al 15\% establecido por normas internacionales y descrito como límite para la adecuada validación de un procedimiento analítico (Shah y col 1992, Prieto y col 2003).

Triclabendazol. El único metabolito de TCBZ encontrado en los tejidos analizados fue $\mathrm{TCBZSO}_{2}$, siendo utilizado este metabolito como residuo marcador. Se ha demostrado que el TCBZ es rápida y completamente metabolizado por oxidación microsomal hepática, siendo biotransformado hacia los metabolitos sulfóxido y sulfona, incrementando su polaridad y facilitando su excreción urinaria y biliar, por lo cual el fármaco original (TCBZ) no es detectado en los tejidos (Hennessy y col 1987).

La especificidad del método queda demostrada por la presencia de extractos limpios, con buena simetría de los picos cromatográficos. Los cromatogramas característicos de la solución patrón puro de $10 \mu \mathrm{g}$ de $\mathrm{TCBZSO}_{2}$ (externo) y los cromatogramas de hígado blanco y las muestras sobrecargadas con $10 \mu \mathrm{g} / \mathrm{g}$ de $\mathrm{TCBZSO}_{2}$ (interno) se presentan en la figura 2.
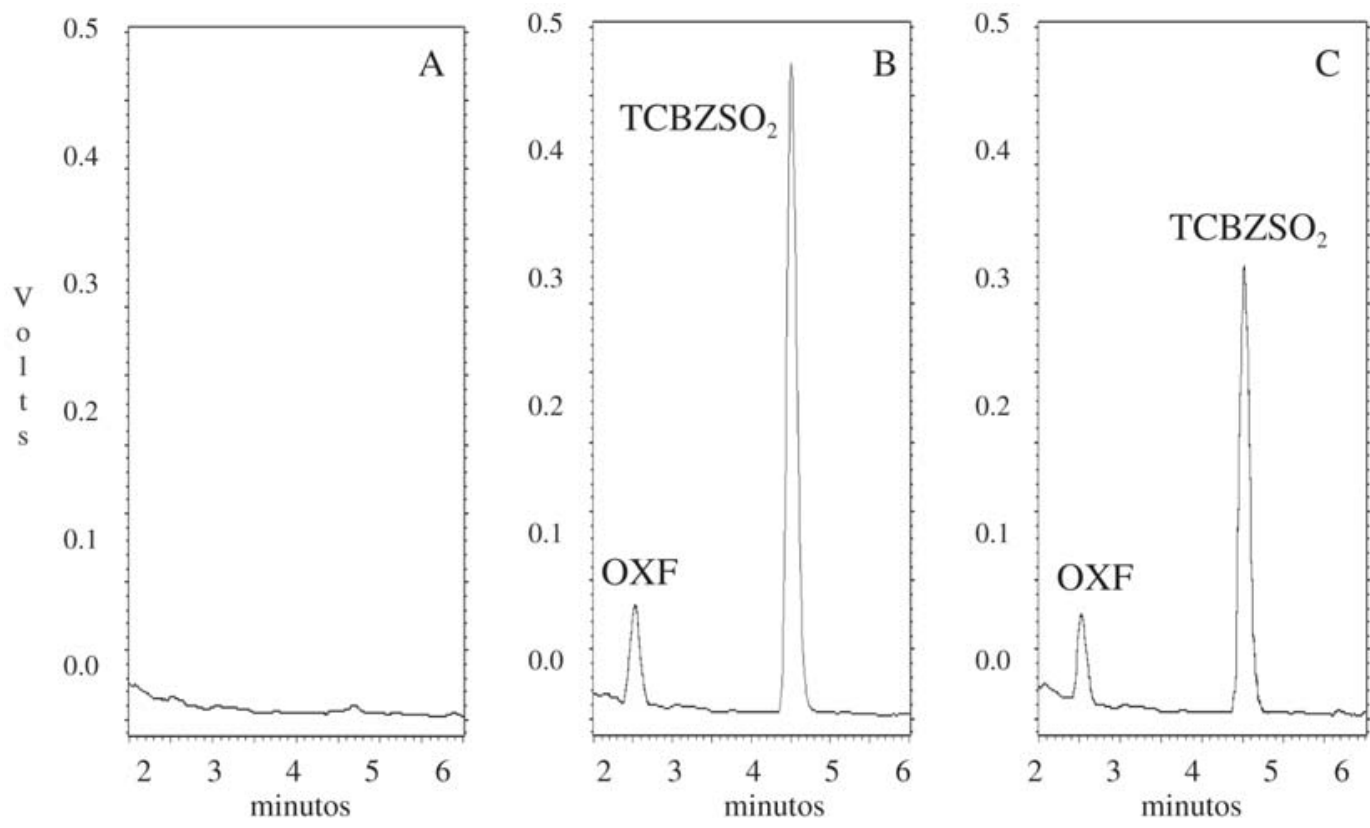

Figura 2. Cromatograma blanco de hígado bovino (A). Cromatograma típico de solución patrón de OXF como estándar interno $(1 \mu \mathrm{g})$ y $\mathrm{TCBZSO}_{2}(10 \mu \mathrm{g})(\mathrm{B})$. Cromatograma típico en hígado de bovino sobrecargado con $10 \mu \mathrm{g} / \mathrm{g}$ de triclabendazol sulfona $\left(\mathrm{TCBZSO}_{2}\right)(\mathrm{C})$. El tiempo de retención fue 2,5 minutos para oxfendazol y 4,5 minutos para triclabendazol sulfona.

Chromatogram of bovine liver blank (A). Typical chromatogram of a solution of oxfendazole (OXF) as internal standard $(1 \mu \mathrm{g})$ and $\mathrm{TCBZSO}_{2}(10 \mu \mathrm{g})(\mathrm{B})$. Chromatogram of bovine liver fortified with $10 \mu \mathrm{g} / \mathrm{g}$ of triclabendazole sulphone $\left(\mathrm{TCBZSO}_{2}\right)(\mathrm{C})$. The retention time was 2,5 minutes for oxfendazole and 4,5 minutes for triclabendazole sulphone. 
Cuadro 2. Parámetros de validación de la metodología analítica para la determinación de triclabendazol sulfona $\left(\mathrm{TCBZ} \mathrm{SO}_{2}\right)$ en tejidos comestibles de bovino.

Analytical methodology validation for the determination of triclabendazole sulphone concentrations $\left(\mathrm{TCBZ} \mathrm{SO}_{2}\right)$ in edible bovine tissues.

\begin{tabular}{|c|c|c|c|c|}
\hline \multirow[b]{2}{*}{$\begin{array}{l}\text { Parámetros de } \\
\text { validación }\end{array}$} & \multicolumn{4}{|c|}{ Tejidos } \\
\hline & Hígado & Riñón & Músculo & Grasa \\
\hline $\begin{array}{l}\text { Porcentaje de } \\
\text { recuperación }(\%)\end{array}$ & 70,00 & 85,11 & 71,25 & 70,00 \\
\hline Linealidad $\left(r^{2}\right)$ & 0,9926 & 0,9965 & 0,9973 & 0,9966 \\
\hline Precisión (C.V.) (\%) & 6,298 & 8,103 & 7,632 & 9,917 \\
\hline Exactitud (E.M.) (\%) & 5,891 & 3,732 & 4,112 & 5,686 \\
\hline $\begin{array}{l}\text { Límite de } \\
\text { cuantificación }(\mu \mathrm{g} / \mathrm{g})\end{array}$ & 0,169 & 0,223 & 0,143 & 0,076 \\
\hline $\begin{array}{l}\text { Límite de } \\
\text { detección }(\mu \mathrm{g} / \mathrm{g})\end{array}$ & 0,086 & 0,097 & 0,110 & 0,032 \\
\hline
\end{tabular}

$\mathrm{r}^{2}$ : coeficiente de determinación; \%: porcentaje; C.V.: coeficiente de variación; E.M.: error medio; ng/g: nanogramos por gramo.

Los porcentajes de recuperación de $\mathrm{TCBZSO}_{2}$ en los diferentes tejidos analizados se presentaron dentro de los rangos de recuperación considerados aceptables $(60$ y $115 \%)$ para métodos de extracción en matrices complejas como las analizadas en este estudio (Ali y col 2000).

Análisis de residuos en bovinos tratados con la asociación de abamectina-triclabendazol vía oral. El Comité de Productos Medicinales Veterinarios de la Agencia Europea para la Evaluación de Productos Medicinales (EMEA 1998), y la Comisión Codex Alimentarius (1995) definen el límite máximo de residuos (LMR) como la concentración máxima de residuos resultantes del uso de un medicamento que se permite legalmente o se reconoce como admisible en un alimento y que no produce riesgos toxicológicos para la salud humana. De acuerdo a ello, se han establecido los valores de límites máximos de residuos (LMR) para los fármacos en estudio; que fueron de $20 \mu \mathrm{g} / \mathrm{kg}$ para abamectina ${ }^{1}$ y de $0,15 \mathrm{mg} / \mathrm{kg}$ para la suma de triclabendazol ${ }^{2}$ y sus metabolitos.

Las concentraciones residuales de ABM y triclabendazol sulfona $\left(\mathrm{TCBZSO}_{2}\right)$ único metabolito de TCBZ detectado en las muestras de tejido son presentadas en los cuadros 3 y 4 respectivamente.

Abamectina. Las mayores concentraciones de ABM se obtuvieron en las muestras de hígado a los 7 días postadministración, seguido del tejido graso, riñón y final-

EMEA, 2000. Abamectin. Summary Report (1).

EMEA, 1998. Triclabendazole. Summary Report.
Cuadro 3. Concentraciones promedio de abamectina (ABM) en hígado, grasa, riñón y músculo de bovinos tratados con la asociación abamectina-triclabendazol (Sofomax ${ }^{\circledR}$ ) determinadas a los 7 y 14 días postratamiento (promedio \pm desviación estándar).

Mean concentrations of Abamectin (ABM) in liver, fat, kidney and muscle of bovines treated with the association abamectintriclabendazole (Sofomax ${ }^{\circledR}$ ) measured at 7 and 14 days post-treatment (mean \pm S.D.).

\begin{tabular}{crrrr}
\hline \multirow{2}{*}{$\begin{array}{c}\text { Días } \\
\text { post-tratamiento }\end{array}$} & \multicolumn{5}{c}{ Tejido } \\
\cline { 2 - 5 } & Hígado & Grasa & Riñón & Músculo \\
\cline { 2 - 5 } 7 días & 4,022 & 2,379 & 0,653 & 0,286 \\
& $\pm 1,644$ & $\pm 1,315$ & $\pm 0,289$ & $\pm 0,293$ \\
\multirow{2}{*}{14 días } & 0,063 & N.D. & N.D. & N.D. \\
& $\pm 0,109$ & & & \\
\hline
\end{tabular}

N.D.: No detectado.

Cuadro 4. Concentraciones promedio de triclabendazol sulfona (TCBZ $\mathrm{SO}_{2}$ ) en hígado, grasa, riñón y músculo de bovinos tratados con la asociación abamectina-triclabendazol (Sofomax ${ }^{\circledR}$ ) determinadas a los 7 y 14 días postratamiento (promedio \pm desviación estándar).

Cuadro 4 Mean concentrations of Triclabendazole sulphone ( $\mathrm{TCBZ} \mathrm{SO}_{2}$ ) in liver, fat, kidney and muscle of bovines treated with the association abamectin-triclabendazole (Sofomax ${ }^{\circledR}$ ) measured at 7 and 14 days post-treatment (mean \pm S.D.).

\begin{tabular}{crrrr}
\hline & \multicolumn{4}{c}{ Concentración de $\mathrm{TCBZ} \mathrm{SO}_{2}(\mu \mathrm{g} / \mathrm{g})$} \\
\cline { 2 - 5 } $\begin{array}{c}\text { Días } \\
\text { post-tratamiento }\end{array}$ & \multicolumn{4}{c}{ Tejido } \\
\cline { 2 - 5 } & Hígado & Grasa & Riñón & Músculo \\
\hline \multirow{2}{*}{7 días } & 0,284 & 0,352 & 0,796 & 0,165 \\
& $\pm 0,081$ & $\pm 0,183$ & $\pm 0,199$ & $\pm 0,053$ \\
\multirow{2}{*}{14 días } & N.D. & N.D. & 0,303 & 0,006 \\
& & & $\pm 0,192$ & $\pm 0,010$ \\
\hline
\end{tabular}

N.D.: No detectado.

mente músculo (cuadro 3). Estos hallazgos coinciden con los estudios realizados por Chiu y Lu (1989) para IVM y con los descritos por Lifschitz y col (2000) para MXD. La lipofilicidad de ABM le confiere una alta afinidad por el tejido hepático y graso, siendo el hígado el último tejido en depurarse de residuos de fármacos de características químicas similares a ABM (Tway y col 1981).

Las concentraciones promedio de ABM observadas en el presente estudio son inferiores a los descritos por Chiu y Lu (1989) en bovinos tratados con $0,3 \mathrm{mg} / \mathrm{kg}$ de ivermectina vía subcutánea. Estas diferencias se pueden explicar por la acción de variables que afectan la biodisponibilidad del fármaco como son el efecto que ejercen la formulación farmacéutica y la vía de administración sobre la absorción (Lanusse 1994). Las bajas concentraciones de ABM observadas en este estudio se deben al hecho de que se utilizó una formulación comercial en forma de suspensión que contenía la asociación 
de ABM-TCBZ, la que fue administrada por vía oral a los bovinos. Estos resultados se corroboran con los bajos niveles de concentración plasmática máxima $(2,5 \mathrm{ng} / \mathrm{mL})$ y de área bajo la curva $(235,4 \mathrm{ng} * \mathrm{~h} / \mathrm{mL})$ descritos en un estudio paralelo sobre la cinética plasmática de estos fármacos (Pérez y col 2001), demostrándose un bajo nivel de absorción de ABM desde el tracto gastrointestinal, lo que se ha atribuido al hecho de que el fármaco se adhiere fuertemente a las partículas de alimento, lo que limita la absorción y determina una menor concentración de ABM en el plasma y los tejidos (McKellar y Benchaoui 1996).

En el presente estudio no se registraron concentraciones detectables de ABM en grasa, riñón y músculo a los 14, 28 y 42 días postratamiento, a diferencia de lo descrito por Chiu y Lu (1989). La EMEA establece un límite máximo de residuos (LMR) de $20 \mu \mathrm{g} / \mathrm{kg}$ de avermectina $\mathrm{B}^{1} \mathrm{a}^{1}(\mathrm{ABM})$ para hígado, equivalente a $20 \mathrm{ng} / \mathrm{g}$ de tejido. Este valor sirve de referencia para el cálculo de los períodos de resguardo del fármaco en carne y tejidos comestibles, los cuales varían de acuerdo a la especie animal de que se trate. En casos en los cuales el hígado no esté disponible, se debe considerar la grasa para el análisis de los metabolitos marcadores ${ }^{1}$. Sin embargo, en este estudio, los niveles residuales de ABM fueron inferiores al LMR establecido para este analito.

Triclabendazol. El metabolito marcador $\mathrm{TCBZSO}_{2}$ fue encontrado en todos los tejidos analizados a los 7 días postratamiento, obteniéndose las mayores concentraciones en las muestras de riñón, seguido de grasa, hígado y músculo (cuadro 4).

La ausencia de niveles detectables del metabolito $\mathrm{TCBZSO}_{2}$ en tejidos posterior a los 14 días de administrado el fármaco se relaciona con su corta duración en el plasma, donde $\mathrm{TCBZSO}_{2}$ permanece solo hasta los 16 días postratamiento (Pérez y col 2001).

No existen referencias acerca de las concentraciones tisulares de TCBZ y sus metabolitos sulfóxido y sulfona en tejidos comestibles. Sin embargo, se ha establecido un LMR provisional de $0,15 \mathrm{mg} / \mathrm{kg}$ para la suma de los residuos de TCBZ y sus metabolitos en tejidos tales como hígado, riñón y músculo ${ }^{2}$.

Es conveniente destacar que las concentraciones de TCBZ $\mathrm{SO}_{2}$ en riñón se mantienen sobre los valores de LMR a los 14 días postratamiento, lo que indica que el período de resguardo para este fármaco debe ser superior a este tiempo. También llaman la atención los bajos niveles de residuos observados en el músculo cuyas concentraciones a los 7 días son cercanas al LMR. En general, los benzimidazoles, además de sufrir metabolismo de primer paso, tienen un corto período de persistencia en tejidos, lo cual explicaría los bajos niveles encontrados en este estudio. Además, la ausencia de TCBZ en tejidos y plasma indica que este fue completamente metabolizado por el hígado e inmediatamente oxidado a sus metabolitos sulfóxido y sulfona (Hennessy y col 1987).
Finalmente, es posible concluir que la metodología analítica desarrollada para la determinación de ABM y TCBZ permitió obtener una adecuada separación de los analitos desde las matrices biológicas estudiadas (hígado, riñón, grasa y músculo), obteniéndose un alto grado de precisión y exactitud. Los porcentajes de recuperación para ABM fluctuaron entre 78 y $84 \%$, mientras que para $\mathrm{TCBZSO}_{2}$ fueron entre 70 y $85 \%$. Las mayores concentraciones residuales de ABM fueron observadas en las muestras de hígado, las que persisten por un período de 14 días. Estos hallazgos se pueden atribuir a la alta lipofilicidad de ABM que le confiere mayor afinidad por el tejido hepático y graso. En tanto que para TCBZSO la mayor concentración de residuos se presentó en riñón, con un período de persistencia de 14 días, lo que sugiere que la vía urinaria representa una ruta importante de eliminación del TCBZ y sus metabolitos.

\section{RESUMEN}

Se realizó un estudio para determinar los niveles de residuos de ABM y TCBZ en tejidos comestibles de bovinos tratados con la formulación comercial que contiene la asociación de ambos fármacos para administración vía oral en bovinos. Se desarrolló y validó un método analítico mediante cromatografía líquida de alta eficiencia (HPLC) usando detector de fluorescencia para ABM y ultravioleta para TCBZ y sus metabolitos. Se utilizaron 16 bovinos con un peso promedio de $232 \pm 37,5 \mathrm{~kg}$, los cuales fueron tratados con la asociación ABM-TCBZ a una dosis de 0,2 $\mathrm{mg}$ de ABM/kg de peso y $10 \mathrm{mg}$ de TCBZ/kg de peso. Los animales fueron sacrificados en grupos de 3 a 4 animales por semana desde el día 7 hasta el día 42 posterior al tratamiento. Para validar la metodología analítica y elaborar las curvas de calibración, se utilizaron muestras de tejido blanco libre de fármacos. La técnica analítica para la detección de ABM en los tejidos analizados presentó valores de recuperación que fluctuaron entre 78 y $84 \%$, con un límite de cuantificación que fue de $0,14 \mathrm{ng} / \mathrm{g}$ para músculo; $0,52 \mathrm{ng} / \mathrm{g}$ para hígado; $0,55 \mathrm{ng} / \mathrm{g}$ para riñón, y $1,4 \mathrm{ng} / \mathrm{g}$ para las muestras de tejido graso. La metodología analítica utilizada en la detección del metabolito de TCBZ presentó porcentajes de recuperación que van desde 70 a $85 \%$ para $\mathrm{TCBZSO}_{2}$, con un límite de cuantificación del método para este analito de $0,076 \mu \mathrm{g} / \mathrm{g}$ para tejido graso; $0,143 \mu \mathrm{g} / \mathrm{g}$ en músculo; $0,169 \mu \mathrm{g} / \mathrm{g}$ en hígado y $0,223 \mu \mathrm{g} / \mathrm{g}$ en riñón. Las mayores concentraciones residuales de ABM fueron observadas en las muestras de hígado (4,02 ng/g) y se mantuvieron por 14 días. En tanto que para $\mathrm{TCBZSO}_{2}$ las mayores concentraciones residuales se presentaron en el riñón $(0,79 \mu \mathrm{g} / \mathrm{g})$, las que persistieron por un período de 14 días.

\section{AGRADECIMIENTOS}

Al doctor Carlos Lanusse, Laboratorio de Farmacología, Departamento de Fisiotología, Facultad de Ciencias Veterinarias, Universidad Nacional del Centro de la Provincia de Buenos Aires, Tandil, Argentina, por haber proporcionado los patrones de TCBZ, TCBZSO y $\mathrm{TCBZSO}_{2}$ y el estándar interno de OXF.

\section{REFERENCIAS}

Ali MS, T Sung, GE McLeroy and ET Phillippo. 2000. Simultaneous determination of eprinomectin, moxidectin, abamectin, doramectin and ivermectin in beef liver by LC with fluorescence detection. JAOAC Int 83: 31-38. 
Alvinerie, M, JF Sutra, D Capela, P Galtier, A Fernández-Suárez, E Horne and M O'Keeffe. 1996. Matrix solid-phase dispersion technique for the determination of moxidectin in bovine tissues. Analyst, 121, 1469-1472.

Chiu SHL and AYH, Lu. 1989. Metabolism and tissue residues. Chapter 8. En: Campbell, W.C. Editor. Ivermectin and abamectin. Springer-Verlag, NY. Inc.

Codex Alimentarius. 1995. Residuos de Medicamentos Veterinarios. Vol. 3, 60-76.

EMEA. 1998. Guideline on validation of analytical procedures: Methodology. VICH topic GL2: the European Agency for the Evaluation of Medicinal products. Veterinary Evaluation unit. Londres.

FAO/OMS. 1991. Evaluación de ciertos residuos de fármacos de uso veterinario en los alimentos. En: $38^{\circ}$ Informe del Comité Mixto FAO/OMS de Expertos en Aditivos Alimentarios. Ginebra.

Hennessy DR, E Lacey, JW Steel and RK Prichard. 1987. The kinetic of triclabendazole disposition in sheep. $J$ Vet Pharmacol Therap 10, 64-72.

Lanusse CE. 1994. Factores que efectan la biodisponibilidad plasmática y eficacia de fármacos antihelmínticos Arch Med Vet 26, 5-14.

Lanusse CE, LH Gascon and RK Prichard. 1995. Comparative plasma disposition kinetics of albendazole fenbendazole, oxfendazole and their metabolites in adult sheep. J Vet Pharmacol Therap 18, 196203

Lifschitz A, F Imperiale, G Virkel, M Muñoz Cobeñas, N Scherling, R DE Lay and C Lanusse. 2000. Depletion of moxidectin tissue residues in sheep. J Agric Food Chem 48, 6011-6015.

Mc.Kellar and HA Benchaoui. 1996. Avermectins and milbemycins. $J$ Vet Pharmacol Therap 19, 331-351.

Misra SC, G Swain, MR Panda, DN Panda and NB Das Mohapatra. 1987. Efficacy of Fasinex (Ciba-Geigy) against fasciolasis in cattle, buffaloes and goats. Indian Vet J 64, 701-704.
Payne L, MB Hicks and T Wehner. 1995. Determination of abamectin and/or Ivermectin in cattle feces at low parts per billion levels using HPLC with fluorescence detection. J Agric Food Chem 43, 1233-1237.

Pérez R, C Godoy, I Cabezas and C Palma. 2001. Cinética plasmática de la asociación abamectina-triclabendazol administrada vía oral en bovinos En: Resúmenes V Jornadas de Buiatría, Puerto Varas, Chile, Pp 189-190.

Prieto JG, G Merino, M Pulido, E Estévez, AJ Molina, L Vila and AI Alvarez. 2003. Improved LC method to determine ivermectin in plasma. J Pharm Biomed Anal 31, 639-645.

Quattrocchi, OA, SI Abelaira De Andrizzi, RF Laba. 1992. Validación de métodos. En : Introducción a la HPLC, Aplicación y Práctica. Artes Gráficas Farro S.A. (ed) Buenos Aires, Argentina, Pp 301-328.

Roudaut B. 1998. Multiresidue method for the determination of avermectin and moxidectin residues in the liver using HPLC with fluorescence detection. Analyst 123, 2541-2544.

Shah V P, K Midha, S Dighe, I McGilveray, J Skelly, A Yacobi. 1992. Analytical methods validation: Bioavailability, Bioequivalence and pharmacokinetic studies J Pharm Sci 81, 309-312.

Taylor M. 2000. Use of anthelmintics in cattle. In Practice. Pp 290-304

Tway P, JS Wood, and G Downing. 1981. Determination of ivermectin in cattle and sheep tissues using high-performance liquid chromatography with fluorescence detection. J Agric Food Chem 29, 1059-1063.

Wolff K, J Eckert, G Schneiter and H Lutz. 1983. Efficacy of triclabendazole againts Fasciola Hepática in sheep and goats. Vet Parasitol 13, 145-150.

Wu Z, J Li, L Zhu, H Luo and X Xu. 2001. Multi-residue analysis of avermectins in swine liver by immunoaffinity extraction and liquid chromatography-mass spectrometry. J Chromatogr B 755, 361-366. 